\title{
USING THE ANALYTIC HIERARCHY PROCESS IN FRAME-BASED EXPERT SYSTEMS
}

Takao TERANO

Central Research Institute of Electric Power Indestry

1-6-1 Ohtemachi, Chiyoda-kn, Tokyo 100, Japan

\begin{abstract}
Expert systems techniques in Artificial Intelligence give an effective way to implement sophisticated software systems. One of the difficulty to apply expert systems techniques. to decision making is caused by the fact that some of the knowledge intrinsically has uncertain information. This paper proposes a new method which uses the Analytic Hierarchy Process (AHP) for handling uncertain knowledge in frame-based expert systems for classification type decision making problems. The method consists of the following two phases: i) Construct a "part-of" frame-taxonomy for the target problem; Then give consistent scores to each item in the frame by applying the AHP. ii) Describe empirical rules; The rules first evaluate the scores in the frame, then derive a plausible conclusion. The proposed method gives some "rational" measures by means of the Consistency Index or the Consistency Ratio to uncertain knowledge, and furthermore it improves the limitation of the AHP i.e., the difficulty to construct a proper hieranchy and the simple decision criteria based on the additive value functions.
\end{abstract}

\section{INTRODUCTION}

Recent advances in artificial intelligence researches give various way to implement sophisticated software systems. Among them, expert systems techniques play the most significant roles. Expert systems are computer programs designed to represent and apply factual knowledge of specific areas of expertise to solve given problems [1].

The main difference between expert systems and conventional programs lies in the representation method of the knowledge of human experts. In expert systems, the knowledge is explicitly represented and stored in knowledge bases. In conventional programs, it is implicitly coded into procedural statements. One of the difficulty to apply expert systems techniques to decision making is caused by the fact that some of the knowledge intrinsically has uncertain information.

Several theories have been proposed for handling uncertainty in expert systems. Various discussions are found in the literature [2,3]. Bhatnagar, R. K. and Kannal, L. N. state the cases where uncertain information must be handled: $i)$ information is partial; ii) the information is not fully reliable; iii) the representation language is 
inherendy imprecise; and iv) information from multiple sources is conflicting [4]. A practical framework - Hypothetical Matching - was proposed by Chandrasekaran, B. in his Generic Tasks concepts [5,6], or Fuzzy sets [7] are considered to be useful.

These theories certainly give some theoretical bases for uncertainty in expent systems, however, in practice, the techniques derived from these theories are very difficult to apply to real world applications unless the knowledge used in expert systems has been gathered into the knowledge base. In other word, there is no practical method to give proper measures with well-founded bases to uncertain information acquired from human experts. We believe that decision theoretic approaches will be effective in such sitnations.

This paper deals with a practical method for handling uncertain information of the cases iii), iv) in [4]. The method combines the Analytic Hierarchy Process (the AHP) [8] - with frame-based and rule-based knowledge representation and reasoning techniques. This paper first describes the ontline of the proposed method by a simple car-selection problem. Then a practical examples is described to show the effectiveness of the proposed method. It is Dam Gate Diagnosing Advisor, which is a frame-based expert system developed by the author. Finally concluding remarks are given.

\section{COMBINING THE AHP WTH FRAME/RULE-BASED KNOWLEDGE REPRESENTATION AND REASONING TECHNIQUES}

\subsection{Frames and Rules in Expert Systems}

The knowledge of human experts is divided to two kinds: the one based on the physical/conceptual structures of the target problem and the other based on the empirical expertise for problem solving. In frame-based expert systems, the former type of knowledge is represented in a hierarchical structures called frames with some kinds of relations. The latter type of knowledge is usually explicilly represented in prodnction rules or atwached procedures embedded in the frames.

These concepts in expert systems are somewhat similar to the coacepts used in the AHP. A frame structure and production rules in expert systems are comesponding to the problem-subproblem hierarchy and pairwise comparison matrices in the AHP respectively. Both concepts in expert systems and the AHP are considered to represent the expertise in problem solving.

\section{The Features of the Analytic Hierarchy Process}

The AIP hierarchically decomposes a given problem $P$ into its smaller constitwent parts $Q(i)$ and then evaluates the weights $W(i)$ of these sub-problems by pairwise comparison judgements. To solve sub-problems $Q(i)$, the same procedure is recursively performed in each hierarchy. The features of the AHP are summarized as follows:

- The weights represent decision makers' judgement, i.e., heuristics of human experts and the eigen value represents its consistency measure within the hierarchy. The feature is useful for giving consistent measures to uncertain information acquired from buman experts.

- The evaluated weights presumes that the problem can be solved by the simple 
linear function based on the additive value theory. If the assumption is violated, the solution of the problem looses its rationality, even the eigenvalue suggests the consistency.

\subsection{Outline of the Proposed Method}

The procedure for applying the AHP only supports the estimation methods by a particular additive value function: This limits the applicability of the method. To improve the defects, We combine the AHP with frame-based and rule-based approaches.

First, note that the concept of frame taxonomy is a natural way to decompose a given problem into some hierarchical structures, which are common in various engineering systems with complex sub-components. Then, we extend the original evaluation method of the AHP in the following ways:

- We give some numerical scores to the basic iteins (leaf items) in the frame. When the basic items have qualitative or non-numerical attributes to be evaluated, these nonnumerical values must be converted to normalized numerical ones. The scores represent the status or conditions of the basic components of the given problem.

- We allow the method to use minimum and/or maximum operators while evaluating the values from the lower hierarchy. Both these operators and the original evaluation function play a role of resolving the conflicts among the values from multiple sources.

- We also allow the method to use production rules to evaluate the sub-problems, that is, typical resoning techniques can be applied to make decisions. The meanings of words in the rules can be determined through the evaluation processes of each subproblem.

These extensions make the AHP more flexible and useful to be applied to expert systems. The proposed method consists of the following two phases:

i) Apply the AHP to small sub-problems:

(i-1) Construct-an appropriate part-of frame-taxonomy for the target problem

Construct hierarchical structures of the target system in the frame-based knowledge representation. The structure is used to evaluate the weights of each items by the AHP.

(i-2) Give the consistent scores to the lower items in the frame

Compute the weights of the lower items by the AHP.

(i-3) Checked the consistency of the weights

Check the consistency of the weights. While there exists inconsistency among the. weights, reconstruct the hierarchy; or retry the pair-wise comparison judgements. ii) Apply production rules for decision making:

(ii-1) Describe the empirical knowledge by production rules

Describe the rules of the empirical knowledge for decision making. The' words used in the rules may be imprecise, because their strict meanings are determined by the results of the estimations of the AHP.

(ii-2) Apply the rules;

Combining the items in the frame with the rules, these rules first evaluate the scores in the frame, then derive a "plausible" conclusion by usual reasoning techniques. 
The above procedure gives some "rational" measures by means of the Consistency Index or the Consistency Ratio to empirical knowledge with uncertainty, and furthermore it improves the limitation of the AHP in the following ways:

(1) The difficulty to develop the proper hierarchy for the given problem:

it is resolved by using the frame taxonomy representation of the target problem.

(2) The simple decision criteria based on the additive value functions of the AHP:

The chain of the reasoning gives the more flexible way for the problem solving.

\subsection{Example of A Car Selection Problem}

In this section, a simple car selection problem is described to illustrate the proposed method. The problem is originally introduced in a Japanese textbook on the AHP [9]. Before applying the proposed method, we will solve the problem only by the AHP and only by the usual frame-/rule-based techniques. Comparing the three methods, we can clarify the features of the proposed method. The problem is to select the best car among $A, B$, and $C$ from the four viewpoints or the attributes of the car, i.e., the cost, the fuel-consumption, the comfort-to-drive and the car-class.

To solve the problem only by the AHP, we will follow the three steps in Figure 1. First, the problem is decomposed into a hierarchical structures (Figure 1 (1)), then the domain experts define the weights of the cars from each attribute by pairwise comparison (Figure 1 (2)). Finally users evaluate each attribute by pairwise comparison (Figure 1 (1)). These results are combined by the additive value function, then give the final conclusion.
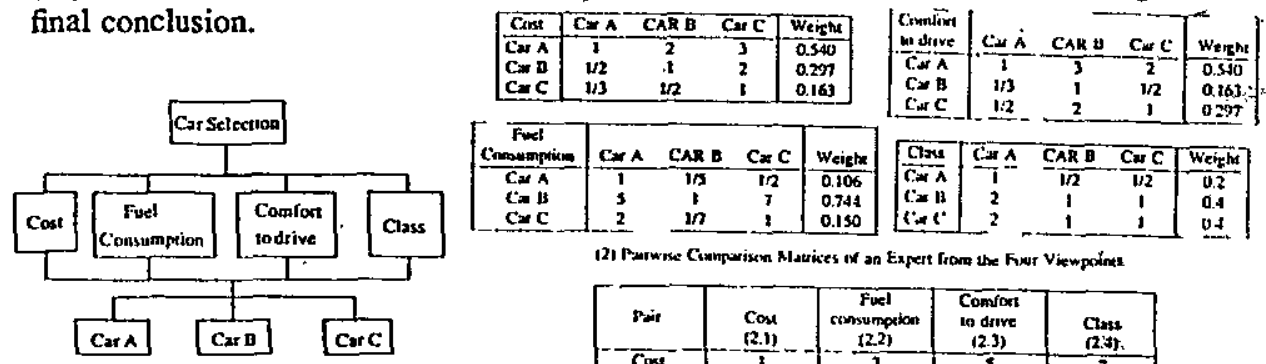

(I) Herarticat Sinucture of the problest

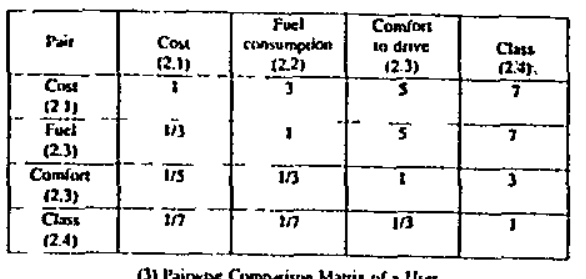

(3) l'airutse Comparison Martin of a User

Figure 1. Car Selection by the AHP

Using the usual frame-/rule-based techniques, the problem is formulated to a typical classification problem. In this case, the original problem is extended in the sense that the attribute of the comfort-to-drive is divided into two sub-attributes, i.e., highspeed and low-speed, because of featuring the expert systems' techniques. The steps for solving the problem are shown in Figure 2. First, the concepts of a car are represented in a prototype car frame, which has four slots., i.e., Cost, Fuel Consumption, Comfort to drive (High/Low speed), and Class. The car A, B, and C are represented in three instance frames with corresponding slot values, e.g., low, 
moderate, or high for the Cost slot, which should be filled by human experts (Figure 2 (1)). Then, the rules for evaluating the comfort-to-drive slot values and the rules for car selection are described. Since these rules represent uncertain knowledge of human experts, they have the $C F$ values to handle the uncertainty (Figure 2 (2)). So far as we use the usual rule based reasoning techniques with uncertainty, It usually takes very long time to adjust the CF values in order to get a plausible conclusion.

Car-Frame
Cost(Evaluation)
Fuel Consumption(Evaluntion)
Comafort to Drive(Evaluation)
High speed(Evaluation)
Low speed(Evaluation)
Class(Evaluation)

\begin{tabular}{|c|c|c|}
\hline $\begin{array}{l}\text { Cins A } \\
\text { low } \\
\text { bad } \\
\text { good } \\
\text { good } \\
\text { good }\end{array}$ & $\begin{array}{l}\text { Car B } \\
\text { moderate } \\
\text { good } \\
\text { bad } \\
\text { bad } \\
\text { bad }\end{array}$ & $\begin{array}{l}\text { Car } \mathrm{C} \\
\text { high } \\
\text { bad } \\
\text { moderate } \\
\text { good } \\
\text { bad }\end{array}$ \\
\hline
\end{tabular}

- Rules for evaluating the slot values

IF comfort-to-drive.high-speed of "car - 'good' AND comfort-to-drive. low-speed of "car $=$ ' good' THEN *comfort-10-drive of "car := 'good' [CF 0.9]

IF comfort-to-drive.high-speed of "car = 'good" AND comfort-lo-drive.low-speed of "car = "bad"

TIEN "comfort-10-drive of "car := "moderate' [CF 0.9]

IF comfort-to-drive.high-speed of "car = 'bad' AND comfort-to-drive.low-speed of "car = "bad"

THEN "comiort-to-drive of "car := "bad" ICF 0.7 !
(1) Car-Frame

IF fuel-consumption is importan

Rules for decision making THEN select the "car which fuel-consumption $=$ 'good' [CF 0.8]

IF cost is important

THEN select the "Car which cost a "low' 1CF 0.91

IF fuel-consumption is importzat AND class is important THEN select the "car which comfort 10 -drive $>$ 'bad' AND class 0 'bad' [CF 0.6]

(2) Selection Rules

Figure 2. Car Selection by Frames and Rules

\begin{tabular}{|c|c|c|c|}
\hline \multicolumn{3}{|c|}{$\begin{array}{l}\text { Cur.firame } \\
\text { Coml(Weight Evaluation) } \\
\text { Fuel Cursumiption(Weight Evaluation) } \\
\text { Cisnfort fu drive(Weight Evaluation) } \\
\text { High speed(Weiglt Evaluation) } \\
\text { Low sperd(Weight Evaluation) } \\
\text { Cluss(Weight Evaluation) }\end{array}$} & $\begin{array}{l}\text { Car A } \\
(0.54 \text { low) } \\
(0.11 \text { bad) } \\
(0.54 \text { good } \\
\text { ("0.95' go } \\
\text { (0.55" go } \\
(0.20 \text { bad) } \\
\text { Car. Fram }\end{array}$ \\
\hline \multicolumn{4}{|c|}{ Weight for comfort-to-drive } \\
\hline & Low speed & high speed & weight \\
\hline $\begin{array}{l}\text { high speed } \\
\text { low speed }\end{array}$ & $1 / 4$ & $\begin{array}{l}4 \\
1\end{array}$ & $\begin{array}{l}0.8 \\
0.2 \\
\end{array}$ \\
\hline
\end{tabular}

chalusulun of contfort lor-drive :- $0.8{ }^{\circ}$ high-speed $+0.2 \cdot$ low-speed

(3) Paitwise Comparison Mtatrix and Evaluation

Formuta of COMIFORT-TO-DRIVE

\begin{tabular}{|c|c|}
\hline $\begin{array}{l}.30 \text { moderale) } \\
.7^{4} \text { good) } \\
.16 \text { bad) } \\
0.20^{\circ} \text { bad) } \\
0.24 \text { bad) }\end{array}$ & 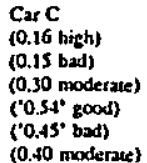 \\
\hline
\end{tabular}

(0.40 moderate) $\quad(0.40$ moderate $)$

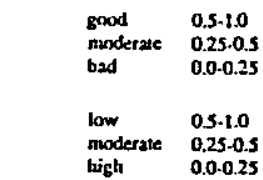

(2) Imetpretation Tabic or Weights

IF fucl-consumption is important

TIIEN select the "car whose fuel-consumption is "good"

IF cost is important

TIIEN select the "car which cost is "good"

If fuel-consumption is impoutant AND class is important "TIIt:N sclect the "cas whath cemfort-2o-drive is NOT' 'bad" ANI class is NOT 'busl'

(4) Car Selection Rules

Figure 3. Car Selection by Frames, Rules and the AHP

In the proposed method, the problem is formulated by both the frame/rule-based knowledge representation and the AHP, which is shown in Figure 3. The frame structure shown in Figure 3 (1) is as same as the one in Figure 2 (1), however, the slot values are different. In this case, each numerical value is determined by the AHP, that is, the values mean the weights of the atributes of the car $A, B$, and $C$, which represent the judgements of human experts. These numerical values are translated into qualitative values such as low, moderate, high, etc., by using the interpretation tables shown in Figure 3 (2). For, example, the values of the Cost slots (0.54 low) of A, ( 0.30 moderate) of $\mathrm{B}$, and ( $0.16 \mathrm{high})$ of $\mathrm{C}$ represent the results of the evaluation by 
the AHP and translated qualitative values. The empirical knowledge of human experts for the small sub-problems is implicitly represented in both the weights and the translation tables. The user evaluates the attributes of the comfort-to-drive of the car by the AHP. The results are shown in Figure 3 (3), which correspond to the rules for evaluating the comfort-to-drive siots in Figure 2 (2). Final decision making rules shown in Figure 3 (3) are similar to the ones in Figure 2 (2). However these rules have no $C F$ values, because the uncertainty in the empirical knowledge is already resolved into the sub-problems, which are solved by the AHP.

\section{A PRACTICAL EXAMPLE OF THE METHOD}

\subsection{Dam Gate Diagnosing Advisor}

Dam gates are one of the most important steel structures at hydro power stations. There are over 1,300 dam gates in Japan, however, the number of human experts who can precisely diagnose them are very small and there are no standards nor authorized manuals established for the purpose.

Dam Gate Diagnosing Advisor (DGDA) is a frame-based expert system for diagnosing dam gates at hydro power stations[10]. The function of DGDA is to diagnose the current status of each dam gate and to predict its remaining life termi based on both structural engineering and empirical standpoints.

To make the diagnoses reliable, we developed an easy-to-use structural analysis program by finite element method and a flexible relational database on questionnaire surveys, measurement data and design information on each dam gate. DGDA is to be used as a front-end system of these conventional systems.

\subsection{Applying the Method}

To diagnose dam'gates, we must use both qualitative questionnaire survey data and empirical knowledge. Because such data and knowledge are subjective, that is, intrinsically uncertain, we apply the proposed method for handling such uncertainty. First, the qualitative field data of the dam gate are converted to numerical values, then evaluated by the AHP. Second, DGDA diagnoses the current status of a dam gate by rule based reasoning, then gets final decisions.

The qualitative data, which should be evaluated by the AHP, are shown in Figure 4. The taxonomy of the data is determined through the design process of DGDA and is coincides with the frame structure in the knowledge base. Each basic item has the value $0,1,2$, or 3 , whose corresponding meanings are "good" to "bad" of the observed condition of the components described in the questionaires of each dam site. Table 1 shows the weights of each component derived from the AHP.

The estimated values of higher level components are interpreted by rules to make a final decision making. For example, the values in Table 1 are used in the following simplified rule:

IF the Appearance is Good, the Operation Condition is Bad,

AND Years of Use is less than 40 Years

THEN the Dam Gate must be Repaired, 
where, the meanings of the words "Appearance", "Operation Condition", and "Years of Use" are defined in the frame taxonomy, and the value of the words are determined from Table 1, and the meanings of the words "Good" and "Bad" is determined by the following criterions:

$\begin{array}{ll}\text { Good: } & \text { score } 0.0-1.0 \\ \text { Fairly Good: } & \text { score } 1.0-1.5 \\ \text { Not Good: } & \text { score } 1.5-2.0 \\ \text { Bad: } & \text { score } 2.0-3.0\end{array}$

Thus, using this method, we have developed the diagnosing expert system to process complex uncertain information without conventional Certainty-Factor-based approaches.

Table 1. Evaluation of Qualitative Data by the AIIP

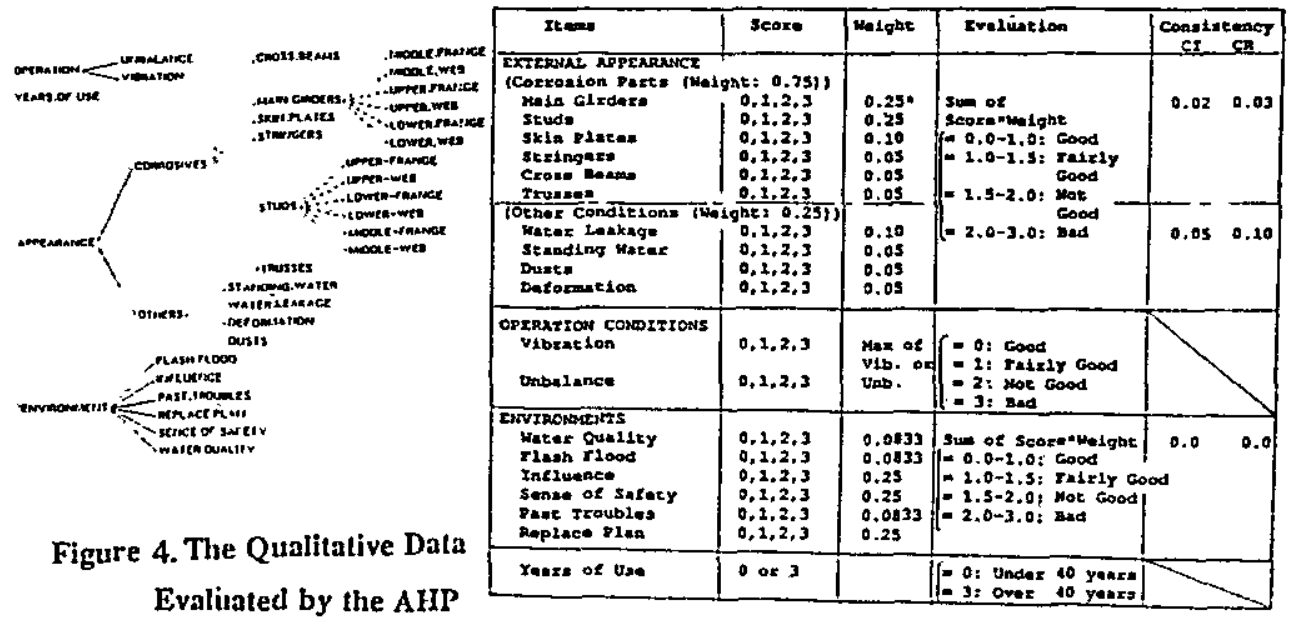

\section{CONCLUDING REMARKS}

This paper proposes a practical method for processing uncertain information using the concepts of the AHP combined with rule-based and frame-based knowledge representation and reasoning techniques. The method is one of the easiest ways to use decision theory to handle uncertainty, and it is especially useful where the knowledge base are managed in the frame based hierarchies.

There are several related works with this paper. Langlotz, C. P. et al. emphasize the importance of the approaches for justifying heuristics [11]. Henrion, M., et al. discuss the results of an experimental comparison of knowledge engineering for expert systems and-for decision making [12]. They state that both expert systems and decision making approaches are useful for developing complex diagnosing. systems. Boose, J.H., et al. report an integrated knowledge acquisition support tool AQUINAS [13]. Although they mention that the AHP is used in AQUINAS for handling uncertain 
information, the detailed discussions are not found in the literature.

Our future plans is to develop more advanced methods based on more complicated decision theoretic models such as the Multi-attribute Utility Function. These approaches will be useful to represent human models in decision making for expert systems.

\section{Acknowledgements}

The author wishes to express their gratitude to the members of ICOT WG on KAS (Knowledge Acquisition System) and PGF (Power Generation Facilities) for their discussions. He would like to express his grateful thanks to Prof. Manabe and Prof: Tone for their discussions on the AHP and to Prof. E. A. Feigentbaum and Dr. P. H. Nii for their discussions on DGDA.

\section{References}

1. Waterman, D. A:; A Guide to Expert Systems, Addison-WèsIeyy; 1985.

2. Gale, W. A. (ed.), Artificial Intelligence and Statistics, Addison-Wesley , 1986.

3. Kanal, L. N. and (eds:); J. F. Lemmer, Uncertainty in Artificial Intelligence, North-Holland, 1986.

4. Bhatnagar, R. K. and Kannal, L. N. "Handling Uncertain Information: A Review of Numeric and Non-Numeric Methods;" in Uncertainty in Artificial Intelligence, ed. L. N: Kanal and J. F. Lemmer, pp. 3-26, North-Holland, 1986.

5. Chandrasekaran, B., "Generic Tasks in Knowledge-Bàsed Reasoning: High Level Building Blocks for Expert System Design," IEEE' Expert, Vol. 1, No. 3, pp. 23-30, FALL 1986.

6. Shortliffe, E. H., Computer-based Medical Consultation: MYCIN, Elsevier, 1976.

7. Zadeh, L. A., "Making Computer Think Like People," IEEE Spectrum, Vol. 21, No. 8, pp. 26-32, Aug. 1984.

8. Saaty, T. L, The Analytic Hierarchy Process - Planning, Priority Setting, Resource Allocation, McGrow-Hill, 1980.

9. Tone, K., The AHP: Game Feeling Decision Aid (in Japanese), Nikkagiren, 1986.

10. Terano, T., Sinohara, Y., Matsui, S., and Nakamura, H., "Dam Gate Diagnosing Advisor - An Expert System for Steel Structures -," Proc. IEEE/SICE Int. Workshop on Artificial Intelligence for Industrial Applications, pp. 129-134, 1988.

11. Langlotz, C. P., Shortliffe, E. H., and Fagan, L. M., "Using Decision Theory to Justify Heuristics," Proc. AAAI 86, pp. 215-219, 1986.

12. Henrion, M. and Cooley, D.R., "An Experimental Comparison of Knowledge Engineering for Expert Systems and for Decision Analysis," Proc. 6th AAAl, pp. 471-476, 1987.

13. Boose, J.H. and Bradshaw, J.M., "Expertise Transfer and Complex Problems: Using AQUINAS as a Knowledge-Acquisition Workbench for Knowledge-Based Systems," Int. J. Man-Machine Studies, Vol. 26, No. 1, pp. 3-28, 1987. 